\title{
Importance Du Couplage De L'inventaire Des Plantes Mellifères Et De L'analyse Pollinique Des Miels De La Saison Des Pluies En Zone Ouest Soudanienne Au Nord-Bénin
}

\author{
Sfich T. B. Ahouandjinou \\ Monique G. Tossou \\ Hounnankpon Yédomonhan \\ Adéline Zanou \\ Aristide C. Adomou \\ Akpovi Akoègninou
}

Laboratoire de Botanique et Ecologie végétale, Faculté des Sciences et Techniques, Université d'Abomey-Calavi, 01 B.P 4521 Cotonou, Bénin

\section{Comlan M. Koudegnan}

Laboratoire de Palynologie, Algologie et Paléoécologie, Facultés des

Sciences, Université de Lomé, Togo

URL:http://dx.doi.org/10.19044/esj.2017.v13n6p38

\begin{abstract}
The melliferous plants vary from one area to another according to biotic, climatic and ecological factors. This study was realized in the Sudanese region of Benin, in order to inventorize honey plants visited by the honeybee Apis mellifera adansonii Latreille. Pollen analysis of 34 samples of honey from three apiaries in the northwest of the country, combined with direct observations around each apiary within $1000 \mathrm{~m}$ radius were realized per month. In total 129 species were censised including 109 species inventorized on the field and 73 taxa identified through pollen analysis. Among these taxa, 43 are identified until species level. The contribution of pollen analysis to the knowledge of melliferous plants is $15.50 \%$. The pollen analysis of honeys and visual inventory of honey plants field are two complementary methods of studying the honey flora.
\end{abstract}

Keywords: Melliferous species, direct obserations, pollen analysis, northern Benin 


\section{Résumé}

Les plantes mellifères varient d'une zone à une autre en fonction des facteurs biotiques, climatiques et écologiques. Cette étude, qui a été réalisée dans la zone soudanienne du Bénin, a pour objectif de recencer les plantes butinées par l'abeille mellifique Apis mellifera adansonii Latreille. A cet effet, une analyse pollinique a été effectuée sur 34 échantillons de miels issus de trois ruchers installés dans le Nord-Ouest du pays. Cette technique d'identification des plantes mellifères a été complétée par des observations directes sur le terrain réalisées dans un rayon de $1000 \mathrm{~m}$ par mois autour de chacun des ruchers. Au total, 129 espèces ont été recensées grâce à ces deux méthodes : 109 espèces sont inventoriées sur le terrain, alors que 73 taxonspolliniques sont identifiés à partir des pollens des miels analysés. Parmi ces taxons polliniques recensés, seuls 43 sont identifés jusqu'au niveau espèce. L'apport de l'analyse pollinique à la connaissance des plantes butinées par les abeilles est de $15,50 \%$. En somme, l'analyse pollinique des miels et l'observation directe sur le terrain représentent donc deux méthodes d'étude complémentaires nécessaires pour un meilleur recensement de la flore mellifère.

Mots clés : Espèces mellifères, observations directes, analyse pollinique, Nord-Bénin

\section{Introduction}

L'apiculture est l'élevage des abeilles en vue de la production du miel. Les plantes mellifères sont des espèces végétales d'où l'abeille prélève des substances, notamment le nectar, le pollen et la résine pour se nourrir et pour élaborer ses produits divers (Nguemo et al., 2004). Il est bien connu que les produits de la ruche reflètent en quantité et en qualité la nature des plantes butinées (Nguemo et al., 2008).

En Afrique, la production de miel constitue une activité importante au sein de l'économie rurale. Au Bénin, l'intérêt qu'accordent les structures de développement local et la population à l'apiculture s'accroît jour après jour (Yedomonhan, 2009a). En effet, l'augmentation de la productivité des ruches et le développement de l'élevage des abeilles domestiques nécessitent la maîtrise des potentialités floristiques et la connaissance des plantes mellifères. Dans ce cadre un programme de recherche sur les plantes mellifères a été initié au Laboratoire de Botanique et Ecologie Végétale depuis l'an 2001. Au cours des activités menées dans le domaine de la botanique apicole, trois mémoires dont deux en zone guinéenne (Fohounfo, 2002 ; Yédomonhan, 2004), un en zone soudano-guinéenne (Dèmènou, 2006); une thèse de Doctorat portant sur les deux zones précitées (Yédomonhan, 2009a) et quatre publications (Tossou 
et al., 2005 ; Yédomonhan et al., 2009b, 2009c ; Tossou et al., 2011) ont été effectués. Ces travaux de recherche menés ne couvrent qu'environ la moitié $\mathrm{du}$ territoire national et les données existantes restent insuffisantes (Yédomonhan, 2009a).

Par ailleurs, les espèces mellifères varient suivant les conditions climatiques (Layens et Bonnier, 1997). Cette situation montre la maîtrise partielle des ressources apicoles du pays. Il urge que les études d'exploration s'étendent en zone soudanienne pour une meilleure connaissance des plantes mellifères. Le présent travail fait suite aux premiers travaux conduits en zones guinéenne et soudano-guinéenne. Son objectif est de recenser les espèces mellifères et de montrer l'importance de la mélissopalynologie dans la connaissance des relations entre les abeilles et les plantes.

\section{Milieu d'etude}

Les études ont été conduites dans la forêt communale de Cobly, dans la forêt classée des collines de Kouandé et dans la zone cynégétique du parc de la Pendjari (Département de l'Atacora) (Figure 1).

Les trois ruchers se localisent entre $10^{\circ}$ et $11^{\circ}$ de latitude Nord et $1^{\circ}$ et $2^{\circ}$ de longitude Est. Le climat est de type soudanien, caractérisé par un régime pluviométrique unimodal. La saison pluvieuse s'étale d'avril à octobre et la saison sèche couvre la période de novembre à mars. Le total pluviométrique annuel est en moyenne de $1229,65 \mathrm{~mm}$. La température moyenne mensuelle oscille entre $29,47^{\circ}$ en août et $38,42^{\circ}$ en mars. Les sols sont de types ferrugineux tropicaux (Faure, 1977). La végétation est composée surtout de savane boisée, de savane arborée et de savane arbustive, de forêt galerie et des lambeaux reliques de forêt dense sèche et de forêt claire (Akoégninou et al., 2006). 


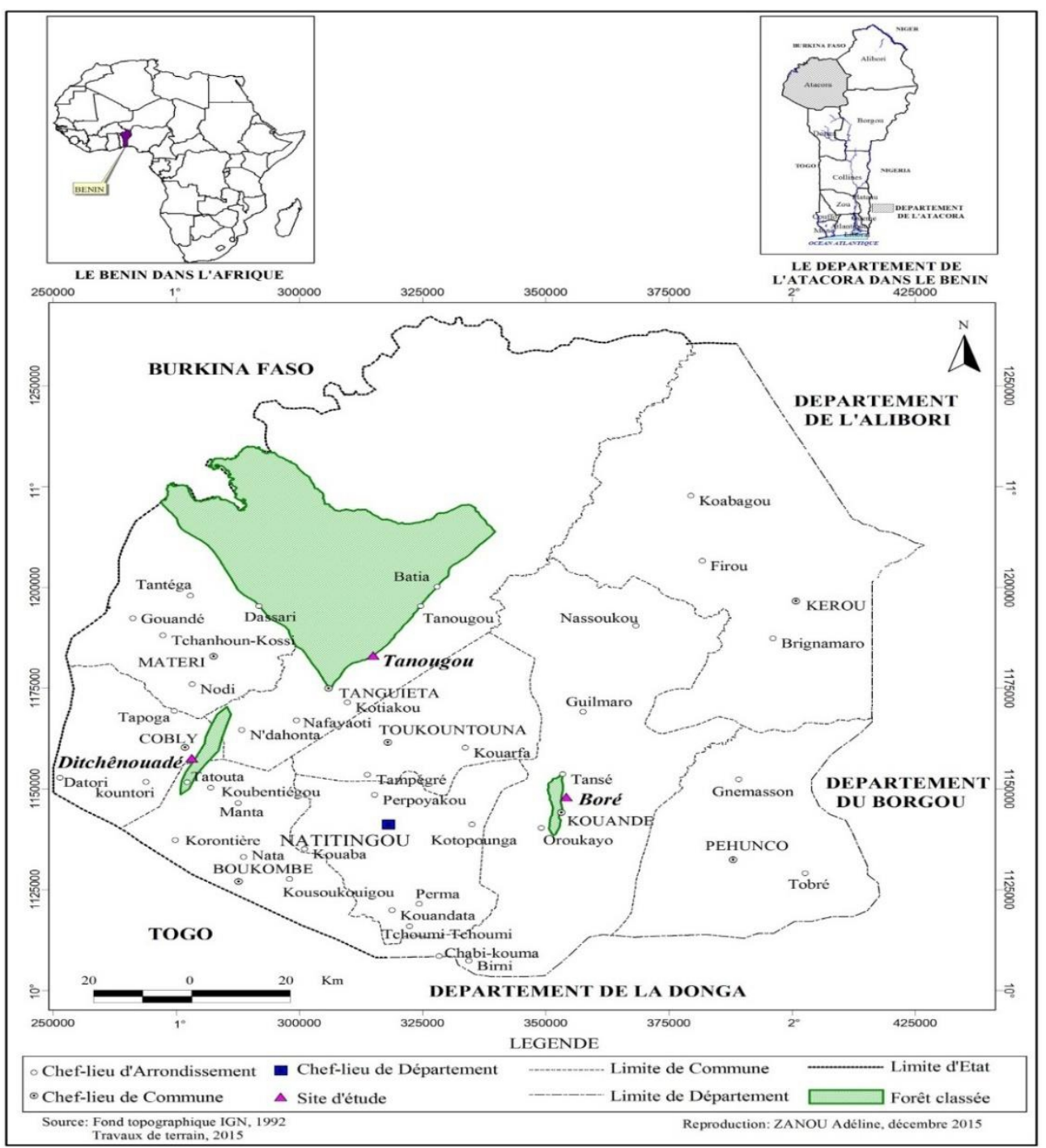

Figure 1: Localisation des sites d'étude dans le département de l'Atacora

\section{Methode d'etude}

\section{Collecte des données}

\section{Observation directe de terrain}

L'observation directe de terrain a été réalisée dans 3 ruchers de 10 ruches kenyanes chacun installés dans 3 différents sites (Figure 1). L'aire d'observation choisie pour ce travail est de $1 \mathrm{~km}$ de rayon autour des ruchers, soit une superficie de $3,14 \mathrm{~km}^{2}$; conforme à celle utilisée par Yédomonhan (2004), Yédomonhan (2009c) et Coulibaly (2013). Cette surface a été divisée en 5 cercles concentriques équidistants de $200 \mathrm{~m}$, qui sont à leur tour subdivisés par 12 rayons de $1 \mathrm{~km}$ formant deux à deux entre eux un angle de 30 degrés (Figure 2). Ainsi, 60 placeaux de $500 \mathrm{~m}^{2}(20 \mathrm{~m} \times 25 \mathrm{~m}$ ) chacun ont 
été installés à tous les points d'intersection des cercles et des rayons (Figure 2).

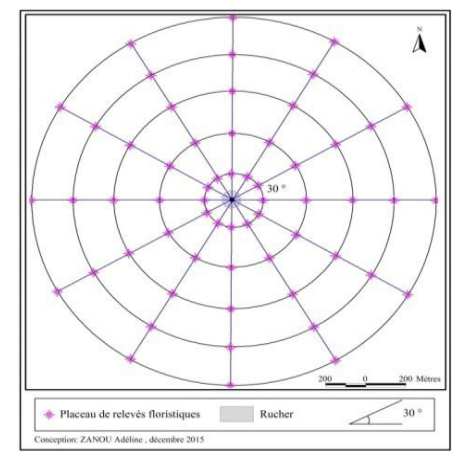

Figure 2 : Répartition des placeaux d'inventaire des plantes mellifères sur le terrain

L'observation directe des plantes a consisté à recenser ou à identifier, durant 4 jours successifs (durée utile pour parcourir la surface d'observation) par mois, toutes les plantes mellifères présentes à l'intérieur des placeaux (Yédomonhan et al., 2009 b). Le taux de butinage d'une espèce butinée a été estimé en pourcentage de fleurs visitées par les abeilles butineuses par rapport à l'ensemble des fleurs de la plante. Les plantes mellifères ont été identifiées sur le terrain et par comparaison aux spécimens de référence de l'Herbier National du Bénin.

\section{Analyse pollinique des miels}

Au total, 34 échantillons de miel récoltés dans les trois ruchers ont été analysés. Leurs lieux de provenance et leur répartition sont consignés dans le tableau 1. L'échantillonnage du miel a été fait seulement au niveau des ruches qui ont été colonisées au cours de la période d'étude dans chaque rucher et plus précisément au niveau des trois derniers rayons formés. Ces nouveaux rayons ne contiennent relativement que du miel produit au cours de la période d'étude.

Tableau 1 : Répartition des échantillons de miels récoltés

\begin{tabular}{|c|c|c|}
\hline $\mathrm{N}^{\circ}$ & Site de récolte & Nombre d'échantillons \\
\hline 1 & Tanguieta & 4 \\
\hline 2 & Cobly & 10 \\
\hline 3 & Kouande & 20 \\
\hline \multicolumn{2}{|c|}{ Total } & 34 \\
\hline
\end{tabular}

L'analyse pollinique a été réalisée par l'unité palynologique du Laboratoire de Botanique et Ecologie Végétale. Le culot contenant les grains de pollens a été extrait par lessivages successifs du melange acidulé obtenu à partir de 20 millilitres de miel prélevés puis traités selon la méthode 
d'acétolyse de Erdtman (1969). L'identification et la numération des pollens ont été effectuées à l'aide d'un microscope de type Olympus ${ }^{\circledR}$ SC35, au grossissement 400x et 1000x. Le comptage est fait par balayage complet de toute la surface de la lamelle. Les déterminations ont été facilitées grâce aux collections de lames de référence et aux ouvrages d'illustrations des pollens de Sowunmi (1973, 1995); Bonnefille et Riollet (1980) et Ybert (1979) disponibles à l'Unité de Palynologie du Laboratoire de Botanique et Ecologie Végétale. Dans l'ensemble, les identifications ont été poussées jusqu'à l'espèce, ou au cas échéant, jusqu'au genre ou famille.

\section{Traitement des données}

\section{Diversité floristique des plantes mellifères}

La diversité spécifique a été évaluée tant pour chaque rucher que pour l'ensemble du milieu d'étude. La nomenclature botanique utilisée est celle de Akoègninou et al. (2006). Le taux de butinage maximal étant de 25\%, les espèces mellifères ayant un taux de butinage d'au moins $10 \%$ sont considérées comme espèce mellifère intensément butinées.

\section{Richesse pollinique et Spectre pollinique}

La richesse pollinique est le nombre total des grains de pollen comptés (Lobreau-Callen et al., 1986). Cette somme pollinique tient compte du nombre de pollens indéterminés, alors que celui des pollens indéterminables est écarté. La richesse pollinique a permis le calcul des taux polliniques et l'obtention du spectre pollinique des échantillons de miel. Le spectre pollinique est constitué de la liste des taxons polliniques identifiés, accompagnés de leurs effectifs et de leur densité relative. Le taxon pollinique est soit l'espèce (par exemple: Tamarindus indica) ou le genre (par exemple: Terminalia-type) ou la famille du grain de pollen identifié suivie de «undiff.» symbolisant les initiaux du mot indifférencié en anglais « undiffentiated » (par exemple: Rubiaceae undiff.).

La densité relative exprime le poids de chaque taxon dans l'affouragement des butineuses ; c'est le quotient en pourcentage de la densité d'un type de pollen par la somme des densités de tous les types de pollens recensés dans l'échantillon. En fonction de cette densité relative des taxons polliniques, quatre catégories de pollen ont été établies suivant la méthode de Feller-Desmaly et Parent (1989). Il s'agit des classes de : pollens dominants (plus de $45 \%$ ), pollens d'accompagnement ou importants (entre 16 et $45 \%$ ), pollens isolés importants (entre 3 et $16 \%$ ) et pollens isolés (moins de $3 \%$ ). L'apport de l'analyse pollinique dans l'inventaire des plantes mellifères est déterminé par comparaison de la diversité spécifique des spectres polliniques et de la flore mellifère obtenue par observation directe du butinage sur le terrain (Lobreau-Callen et Damblon, 1994 ; Tossou et al., 2011). 


\section{Resultats}

\section{Diversité de la flore mellifère de la zone Ouest soudanienne}

La flore mellifère totale inventoriée est de 109 espèces, dont 53 dans la forêt communale de Cobly, 60 dans la forêt classé des collines de Kouandé et 43 dans la zone cynégétique du parc de la Pendjari (Tableau 2). La composition en plantes mellifères varie d'un site d'étude à un autre. Neuf espèces sont communes aux trois sites, soit $8,2 \%$ de la flore mellifère inventoriée. La contribution spécifique en plantes mellifères de chacun des sites est de 22 espèces $(20,1 \%)$ pour la forêt communale de Cobly, 31 espèces $(28,4 \%)$ pour la forêt classée des collines de Kouandé et 18 espèces $(16,5 \%)$ pour la zone cynégétique du parc de la Pendjari.

L'ensemble des plantes butinées par les abeilles identifiées sur les sites d'étude se répartissent en 91 genres et 44 familles. La famille des Leguminosae est la plus visitée avec pour richesse spécifique 23 espèces visitées, soit $21,1 \%$ de l'ensemble des espèces mellifères recensées. Elles sont suivies des Rubiaceae avec 8 espèces (soit 7,3\%), des Asteraceae, des Euphorbiaceae, des Malvaceae et des Verbenaceae avec 5 espèces chacune (soit $4,5 \%$ chacune).

Pour la production du miel, à côté du principal nutriment qu'est le nectar, les abeilles ouvrières récoltent du pollen, de la résine et du jus de fruit. En fonction du nutriment recherché, on note une typologie des plantes qui varie d'un site d'étude à un autre. Ainsi, on peut distinguer des plantes nectarifères (qui ne nourrissent les abeilles uniquement qu'avec du nectar), des plantes pollinifères (uniquement qu'avec les pollens), des plantes nectarifères et pollinifères (à la fois avec le nectar et les pollens). En outre, on a également relevé des plantes sur lesquelles les abeilles butinent à la fois, soit le nectar, le pollen et la résine, ou le nectar, le pollen et le jus de fruit, ou encore le nectar, le pollen, la résine et le jus de fruit et enfin les plantes butinées uniquement pour le jus de fruit (Figures 3, 4 et 5).

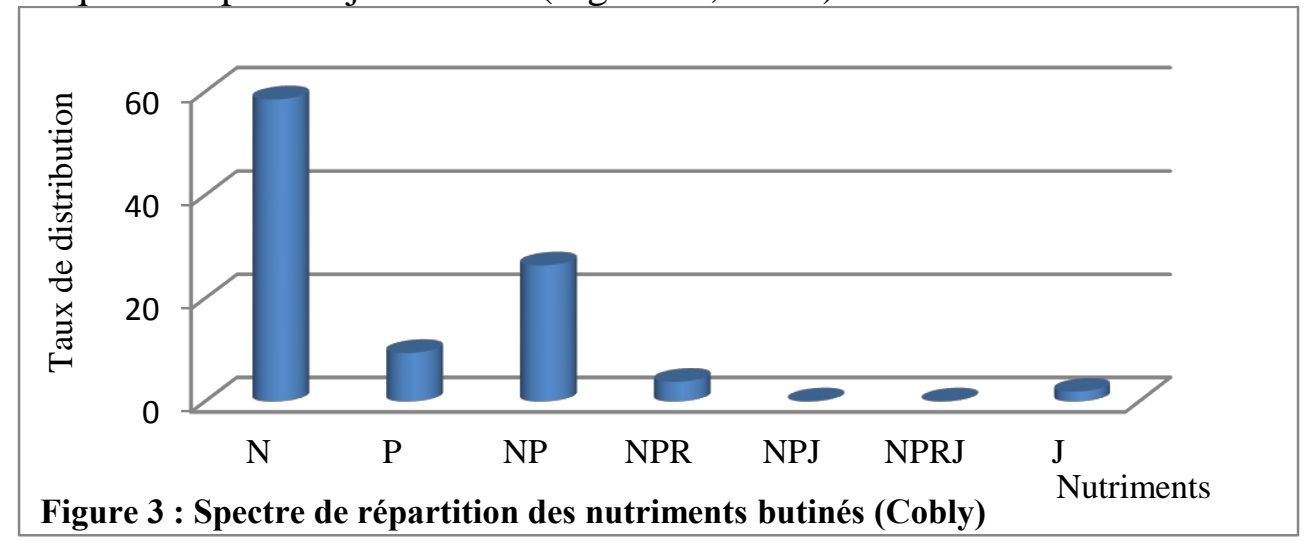



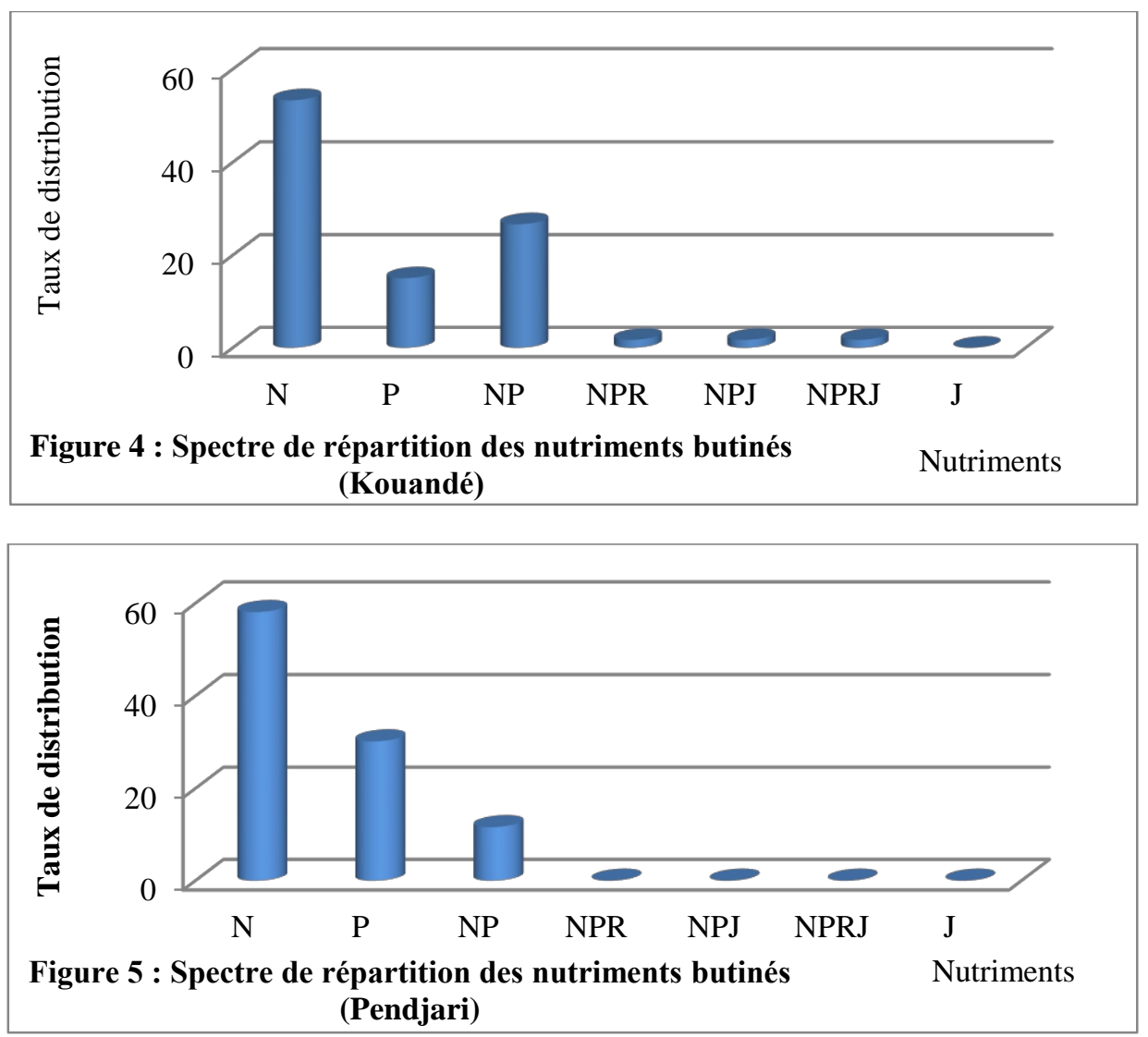

Significations : $\mathrm{N}=$ nectar $; \mathrm{P}=$ pollen $; \mathrm{NP}=$ nectar et pollen $; \mathrm{NPR}=$ nectar, pollen et résine ; $\mathrm{NPJ}=$ nectar, pollen et jus de fruit ; NPRJ $=$ nectar, pollen, résine et jus de fruit ; $\mathrm{J}=$ jus de aux abeilles le même nutriment. Il s'agit de: Anogeissus leiocarpa, Leptadenia hastata, Mitracarpus hirtus et sterculia setigera. Toutefois, chez les 05 autres espèces mellifères restantes, le nutriment produit et butiné par les abeilles diffère d'un site d'étude à un autre. C'est le cas de Bridelia scleroneura et Gardenia erubescens exclusivement nectarifères dans la forêt classée des collines de Kouandé mais qui sont à la fois nectarifères et pollinifères dans la forêt communale de Cobly et dans la zone cynégétique du parc de la Pendjari.

En tenant compte uniquement du taux de butinage, on n'a pu identifier certaines de ces espèces mellifères qui sont intensément butinées : 06 espèces dans la forêt communale de Cobly (Ficus sycomorus, Cyphostemma adenocaule, Feretia apodanthera, Parkia biglobosa, Acacia sieberiana et Vitellaria paradoxa), 03 espèces dans la forêt classée des collines de Kouandé (Anacardium occidentale, Bombax costatum et Vitellaria paradoxa) et 02 espèces dans la zone cynégétique du parc de Pendjari (Acacia dudgeonii et Parkia biglobosa) (Tableau 2). 
Tableau 2 : Liste des espèces mellifères identifiées sur le terrain au cours de la saison pluvieuse avec leur période de floraison optimale

FB : forme biologique (A : arbre, a : arbuste, $\mathrm{H}$ : herbacée) ; Site (C : Cobly, K : Kouandé,

$\mathrm{T}$ : Tanguiéta) ; Nu : nutriment ( $\mathrm{J}$ : jus de fruit, $\mathrm{N}:$ nectar, $\mathrm{P}:$ pollen, $\mathrm{R}:$ résine) ; TB : taux de butinage ; Mois de floraison (A : avril, Mi : mai, J : juin, Ji : juillet, Ao : Août, S : septembre) $;{ }^{*}$ : en fleur à Cobly, - : en fleur à Kouandé, ${ }^{\circ}:$ en fleur à Tanguiéta; espèce mellifère intensément butinée, fr : en fruit.

\begin{tabular}{|c|c|c|c|c|c|c|c|c|c|c|}
\hline \multirow[b]{2}{*}{ Espèces et auteurs } & \multirow{2}{*}{$\begin{array}{l}\mathrm{F} \\
\mathrm{B}\end{array}$} & \multirow[b]{2}{*}{$\begin{array}{c}\text { Sit } \\
\text { e }\end{array}$} & \multirow[b]{2}{*}{$\mathrm{Nu}$} & \multirow{2}{*}{$\begin{array}{l}\mathrm{TB} \\
(\% \\
)\end{array}$} & \multicolumn{6}{|c|}{ Mois de floraison } \\
\hline & & & & & A & $\begin{array}{c}\mathrm{M} \\
\mathrm{i}\end{array}$ & $\mathrm{J}$ & $\mathrm{Ji}$ & $\begin{array}{l}\mathrm{A} \\
\mathrm{O}\end{array}$ & $S$ \\
\hline \multicolumn{11}{|l|}{ Acanthaceae } \\
\hline Justicia insularis T.Anderson & $\mathrm{H}$ & $\mathrm{K}$ & $\mathrm{N}$ & 1 & & & & & & - \\
\hline $\begin{array}{l}\text { Monechma ciliatum (Jacq.) Milne- } \\
\text { Redh. }\end{array}$ & $\mathrm{H}$ & $\mathrm{K}$ & $\mathrm{N}$ & 1 & & & & & - & - \\
\hline \multicolumn{11}{|l|}{ Amaranthaceae } \\
\hline Celosia trigyna $\mathrm{L}$. & $\mathrm{H}$ & $\mathrm{T}$ & $\mathrm{P}$ & $1 / 1$ & & & & & $\circ$ & $\circ$ \\
\hline Gomphrena celosioides Mart. & $\mathrm{H}$ & $\mathrm{T}$ & $\mathrm{P}$ & 1 & & & & & & $\circ$ \\
\hline \multicolumn{11}{|l|}{ Anacardiaceae } \\
\hline Anacardium occidentale L. & $\mathrm{a}$ & $\mathrm{K}$ & $\begin{array}{c}\text { JNP } \\
\mathrm{R}\end{array}$ & 10 & - & & & - & - & \\
\hline Mangifera indica L. & A & $\mathrm{K}$ & JNP & 3 & fr & $\begin{array}{l}\mathrm{f} \\
\mathrm{r}\end{array}$ & & & & \\
\hline \multicolumn{11}{|l|}{ Anthericaceae } \\
\hline $\begin{array}{c}\text { Chlorophytum senegalense (Baker) } \\
\text { Hepper }\end{array}$ & $\mathrm{H}$ & $\begin{array}{l}\mathrm{K} / \\
\mathrm{T}\end{array}$ & $\begin{array}{c}\mathrm{N} / \mathrm{N} \\
\mathrm{P}\end{array}$ & $1 / 1$ & & & & - & $\bar{\circ}$ & \\
\hline \multicolumn{11}{|l|}{ Araliaceae } \\
\hline $\begin{array}{l}\text { Cussonia arborea Hochst. ex A. } \\
\text { Rich. }\end{array}$ & $\mathrm{a}$ & $\mathrm{K}$ & $\mathrm{N}$ & 4 & - & - & - & & & \\
\hline \multicolumn{11}{|l|}{ Asclepiadaceae } \\
\hline Leptadenia hastata (Pers.) Decne. & $\mathrm{L}$ & $\begin{array}{l}\mathrm{C} / \\
\mathrm{K} / \\
\mathrm{T}\end{array}$ & $\begin{array}{l}\mathrm{N} / \mathrm{N} / \\
\mathrm{N}\end{array}$ & $\begin{array}{l}3 / 4 \\
/ 1\end{array}$ & $*$ & - & - & $\begin{array}{l}* \\
\circ\end{array}$ & & \\
\hline \multicolumn{11}{|l|}{ Asteraceae } \\
\hline Ageratum conyzoides $\mathrm{L}$. & $\mathrm{H}$ & $\mathrm{T}$ & $\mathrm{N}$ & 1 & & & & & $\circ$ & c \\
\hline Aspilia bussei O.Hoffm. \& Muschl. & $\mathrm{H}$ & $\mathrm{K}$ & NP & 1 & & & - & - & - & 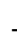 \\
\hline $\begin{array}{c}\text { Aspilia helianthoides (Schumach. } \\
\text { \&Thonn.) } \\
\text { Oliv. \& Hiern }\end{array}$ & $\mathrm{H}$ & $\mathrm{K}$ & NP & 3 & & & & & - & 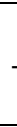 \\
\hline Tithonia diversifolia (Hemsl.) A.Gray & $\mathrm{H}$ & $\mathrm{K}$ & $\mathrm{P}$ & 2 & & & & & & - \\
\hline Tridax procumbens $\mathrm{L}$. & $\mathrm{H}$ & $\mathrm{K}$ & $\mathrm{P}$ & 5 & - & - & - & - & - & - \\
\hline \multicolumn{11}{|l|}{ Bombacaceae } \\
\hline Adansonia digitata $\mathrm{L}$. & A & $\begin{array}{c}\mathrm{K} / \\
\mathrm{T}\end{array}$ & $\begin{array}{c}\mathrm{NP} / \\
\mathrm{N}\end{array}$ & $5 / 1$ & $\circ$ & $\circ$ & $\bar{\circ}$ & $\bar{\circ}$ & - & - \\
\hline
\end{tabular}




\begin{tabular}{|c|c|c|c|c|c|c|c|c|c|c|}
\hline \multirow[b]{2}{*}{ Espèces et auteurs } & \multirow{2}{*}{$\begin{array}{l}\mathrm{F} \\
\mathrm{B}\end{array}$} & \multirow{2}{*}{$\begin{array}{c}\text { Sit } \\
\mathrm{e}\end{array}$} & \multirow[b]{2}{*}{$\mathrm{Nu}$} & \multirow{2}{*}{$\begin{array}{l}\mathrm{TB} \\
(\% \\
)\end{array}$} & \multicolumn{6}{|c|}{ Mois de floraison } \\
\hline & & & & & A & $\begin{array}{l}\mathrm{M} \\
\mathrm{i}\end{array}$ & $\mathrm{J}$ & $\mathrm{Ji}$ & $\begin{array}{c}\mathrm{A} \\
\mathrm{O}\end{array}$ & S \\
\hline Bombax costatum Pellegr. \& Vuillet & A & $\mathrm{K}$ & NP & 10 & & - & & & & \\
\hline \multicolumn{11}{|l|}{ Capparaceae } \\
\hline Cleome gynandra L. & $\mathrm{H}$ & $\begin{array}{l}\mathrm{C} / \\
\mathrm{K}\end{array}$ & $\begin{array}{l}\mathrm{NP} / \\
\mathrm{NP}\end{array}$ & $3 / 1$ & & & & - & $*$ & $*$ \\
\hline Cleome viscosa $\mathrm{L}$. & $\mathrm{H}$ & $\mathrm{T}$ & NP & 2 & & & $\circ$ & $\circ$ & $\circ$ & $\circ$ \\
\hline \multicolumn{11}{|l|}{ Celastraceae } \\
\hline $\begin{array}{l}\text { Gymnosporia senegalensis (Lam.) } \\
\text { Loes. }\end{array}$ & $\mathrm{a}$ & $\mathrm{T}$ & $\mathrm{N}$ & 1 & $\circ$ & $\circ$ & $\circ$ & & & \\
\hline \multicolumn{11}{|l|}{ Chrysobalanaceae } \\
\hline $\begin{array}{l}\text { Parinari curatellifolia Planch. ex } \\
\text { Benth. } \\
\end{array}$ & a & $\begin{array}{c}\mathrm{C} / \\
\mathrm{T}\end{array}$ & $\begin{array}{c}\mathrm{N} / \mathrm{N} \\
\mathrm{P}\end{array}$ & $5 / 4$ & & $\circ$ & $\begin{array}{c}* \\
\circ \\
\end{array}$ & & & \\
\hline \multicolumn{11}{|l|}{ Clusiaceae } \\
\hline $\begin{array}{c}\text { Psorospermum corymbiferum } \\
\text { Hochr.var. } \\
\text { corymbiferum } \\
\end{array}$ & a & $\mathrm{C}$ & $\mathrm{N}$ & 1 & & $*$ & & & & \\
\hline \multicolumn{11}{|l|}{ Cochlospermaceae } \\
\hline Cochlospermum planchoni Hook.f. & $\mathrm{H}$ & $\begin{array}{l}\mathrm{C} / \\
\mathrm{K}\end{array}$ & $\mathrm{P} / \mathrm{P}$ & $5 / 2$ & & & & & & - \\
\hline \multicolumn{11}{|l|}{ Combretaceae } \\
\hline Combretum collinum Fresen. & $\mathrm{a}$ & $\begin{array}{l}\mathrm{C} / \\
\mathrm{T}\end{array}$ & $\mathrm{N} / \mathrm{N}$ & $3 / 2$ & & $\circ$ & $\circ$ & $\begin{array}{l}* \\
\circ \\
\circ\end{array}$ & & \\
\hline Terminalia macroptera Guill. \& Perr. & $\mathrm{a}$ & $\mathrm{C}$ & $\mathrm{N}$ & 2 & $*$ & $*$ & & & & \\
\hline $\begin{array}{l}\text { Anogeissus leiocarpa (DC.) Guill. \& } \\
\text { Perr. }\end{array}$ & A & $\begin{array}{l}\mathrm{C} / \\
\mathrm{K} / \\
\mathrm{T}\end{array}$ & $\begin{array}{l}\mathrm{NP} / \\
\mathrm{NP} / \\
\mathrm{NP}\end{array}$ & $\begin{array}{l}5 / 5 \\
/ 6\end{array}$ & & & & & $\begin{array}{l}* \\
\\
-\end{array}$ & $\begin{array}{l}* \\
- \\
\end{array}$ \\
\hline $\begin{array}{l}\text { Combretum adenogonium Steud. ex } \\
\text { A.Rich. }\end{array}$ & $\mathrm{a}$ & $\begin{array}{l}\mathrm{C} / \\
\mathrm{K}\end{array}$ & $\begin{array}{l}\mathrm{NP} / \\
\mathrm{NP}\end{array}$ & $5 / 3$ & $\begin{array}{l}* \\
- \\
\end{array}$ & & & & & \\
\hline \multicolumn{11}{|l|}{ Commelinaceae } \\
\hline Commelina benghalensis L. & $\mathrm{H}$ & $\begin{array}{l}\mathrm{C} / \\
\mathrm{K}\end{array}$ & $\mathrm{N} / \mathrm{N}$ & $1 / 1$ & & - & - & * & * & $\begin{array}{ll}* \\
*\end{array}$ \\
\hline Commelina erecta L. ssp. erecta & $\mathrm{H}$ & $\mathrm{K}$ & $\mathrm{N}$ & 1 & & & & & - & - \\
\hline Cyanotis lanata Benth. & $\mathrm{H}$ & $\begin{array}{l}\mathrm{C} / \\
\mathrm{K}\end{array}$ & $\mathrm{P} / \mathrm{P}$ & $3 / 3$ & & & & & - & \\
\hline \multicolumn{11}{|l|}{ Convolvulaceae } \\
\hline $\begin{array}{c}\text { Ipomoea beninensis Akoègninou, } \\
\text { Lisowski } \\
\& \text { Sinsin } \\
\end{array}$ & $\mathrm{H}$ & $\mathrm{T}$ & $\mathrm{N}$ & 1 & $\circ$ & $\circ$ & & & & \\
\hline \multicolumn{11}{|l|}{ Dipterocarpaceae } \\
\hline Monotes kerstingii Gilg & $\mathrm{a}$ & $\mathrm{K}$ & $\mathrm{P}$ & 1 & & & & & & - \\
\hline Euphorbiaceae & & & & & & & & & & \\
\hline
\end{tabular}




\begin{tabular}{|c|c|c|c|c|c|c|c|c|c|c|}
\hline \multirow[b]{2}{*}{ Espèces et auteurs } & \multirow{2}{*}{$\begin{array}{l}\mathrm{F} \\
\mathrm{B}\end{array}$} & \multirow{2}{*}{$\begin{array}{c}\text { Sit } \\
\mathrm{e}\end{array}$} & \multirow[b]{2}{*}{$\mathrm{Nu}$} & \multirow{2}{*}{$\begin{array}{c}\mathrm{TB} \\
(\% \\
)\end{array}$} & \multicolumn{6}{|c|}{ Mois de floraison } \\
\hline & & & & & A & $\begin{array}{l}\mathrm{M} \\
\mathrm{i}\end{array}$ & $\mathrm{J}$ & $\mathrm{Ji}$ & $\begin{array}{l}\mathrm{A} \\
\mathrm{O}\end{array}$ & $\mathrm{S}$ \\
\hline Bridelia ferruginea Benth. & $\mathrm{a}$ & $\mathrm{C}$ & $\mathrm{N}$ & 1 & $*$ & & & & & \\
\hline $\begin{array}{l}\text { Margaritaria discoidea (Baill.) } \\
\text { Webster }\end{array}$ & $\mathrm{a}$ & $\mathrm{C}$ & $\mathrm{N}$ & 3 & $*$ & & & & & \\
\hline Bridelia scleroneura Müll.Arg. & $\mathrm{a}$ & $\begin{array}{l}\mathrm{C} / \\
\mathrm{K} / \\
\mathrm{T}\end{array}$ & $\begin{array}{c}\mathrm{NP} / \\
\mathrm{N} / \mathrm{N} \\
\mathrm{P}\end{array}$ & $\begin{array}{l}1 / 1 \\
/ 2\end{array}$ & & & & $\begin{array}{l}* \\
- \\
\circ\end{array}$ & $\begin{array}{l}* \\
\\
\end{array}$ & \\
\hline $\begin{array}{c}\text { Flueggea virosa (Roxb. ex Willd.) } \\
\text { Voigt }\end{array}$ & $\mathrm{a}$ & $\mathrm{T}$ & NP & 5 & & $\circ$ & $\circ$ & $\circ$ & $\circ$ & $\circ$ \\
\hline Euphorbia heterophylla L. & $\mathrm{H}$ & $\mathrm{K}$ & $\mathrm{P}$ & 3 & & & - & - & - & \\
\hline \multicolumn{11}{|l|}{ Flacourtiaceae } \\
\hline Oncoba spinosa Forssk. & $\mathrm{a}$ & $\mathrm{K}$ & NP & 5 & & - & & & & \\
\hline \multicolumn{11}{|l|}{ Lamiaceae } \\
\hline Hoslundia opposita Vahl & $\mathrm{a}$ & $\mathrm{K}$ & $\mathrm{N}$ & 1 & & & & - & - & - \\
\hline Hyptis suaveolens (L.) Poit. & $\mathrm{H}$ & $\begin{array}{l}\mathrm{C} / \\
\mathrm{K}\end{array}$ & $\mathrm{N} / \mathrm{N}$ & $3 / 4$ & & & & & $*$ & - \\
\hline Leucas martinicensis (Jacq.) R.Br. & $\mathrm{H}$ & $\mathrm{C}$ & $\mathrm{N}$ & 1 & & & & & $*$ & $*$ \\
\hline \multicolumn{11}{|l|}{ Leguminosae-Caesalpinioideae } \\
\hline Burkea africana Hook. & $\mathrm{A}$ & $\mathrm{K}$ & $\mathrm{N}$ & 1 & - & & & & & \\
\hline Senna occidentalis (L.) Link & $\mathrm{H}$ & $\mathrm{C}$ & $\mathrm{N}$ & 1 & & & & & $*$ & $*$ \\
\hline Tamarindus indica $\mathrm{L}$. & A & $\begin{array}{l}\mathrm{C} / \\
\mathrm{T}\end{array}$ & $\mathrm{N} / \mathrm{N}$ & $4 / 1$ & $\begin{array}{c}* \\
\\
\end{array}$ & $\begin{array}{c}* \\
\\
\circ\end{array}$ & $*$ & & & \\
\hline $\begin{array}{c}\text { Chamaecrista mimosoides (L.) } \\
\text { Greene }\end{array}$ & $\mathrm{H}$ & $\begin{array}{l}\mathrm{C} / \\
\mathrm{K} / \\
\mathrm{T}\end{array}$ & $\begin{array}{c}\mathrm{N} / \mathrm{P} / \\
\mathrm{N}\end{array}$ & $\begin{array}{l}1 / 2 \\
/ 1\end{array}$ & & & & & $\begin{array}{l}* \\
- \\
\circ \\
\end{array}$ & $\begin{array}{l}* \\
- \\
\circ\end{array}$ \\
\hline Detarium microcarpum Guill. \& Perr. & $\mathrm{a}$ & $\mathrm{C}$ & NP & 3 & & & & & $*$ & $*$ \\
\hline \multicolumn{11}{|l|}{ Leguminosae-Mimosoideae } \\
\hline Entada africana Guill. \& Perr. & $\mathrm{a}$ & $\mathrm{C}$ & $\mathrm{N}$ & 4 & $*$ & $*$ & $*$ & $*$ & & \\
\hline Acacia gourmaensis A.chev. & $\mathrm{a}$ & $\mathrm{C}$ & NP & 5 & $*$ & & & & $*$ & $*$ \\
\hline $\begin{array}{c}\text { Acacia polyacantha Willd. Ssp. } \\
\text { Campylacantha (Hochst. Ex A.Rich.) } \\
\text { Brenan }\end{array}$ & A & $\mathrm{C}$ & NP & 4 & & $*$ & $*$ & $*$ & $*$ & \\
\hline $\begin{array}{c}\text { Dichrostachys cinerea (L.) Wight \& } \\
\text { Arn. }\end{array}$ & $\mathrm{a}$ & $\mathrm{C}$ & NP & 2 & $*$ & $*$ & $*$ & $*$ & $*$ & $*$ \\
\hline $\begin{array}{c}\text { Parkia biglobosa (Jacq.) R. Br. ex } \\
\text { Benth. }\end{array}$ & A & $\begin{array}{l}\mathrm{C} / \\
\mathrm{T}\end{array}$ & $\begin{array}{l}\mathrm{NP} / \\
\mathrm{NP}\end{array}$ & $\begin{array}{l}25 / \\
25\end{array}$ & $\begin{array}{c}* * \\
\circ \\
\end{array}$ & & & & & \\
\hline Acacia Sieberiana DC. Var. villosa & $\mathrm{A}$ & $\mathrm{C}$ & NPR & 10 & $*$ & & & & & \\
\hline Acacia dudgeonii Craib ex Holland & $\mathrm{a}$ & $\begin{array}{l}\mathrm{C} / \\
\mathrm{K} / \\
\mathrm{T}\end{array}$ & $\begin{array}{l}\mathrm{NR} / \\
\mathrm{N} / \mathrm{N}\end{array}$ & $\begin{array}{l}2 / 4 \\
/ 15\end{array}$ & - & $\circ$ & $\begin{array}{l}* \\
\circ\end{array}$ & $*$ & $*$ & \\
\hline Acacia seyal Delile var. seyal & $\mathrm{a}$ & $\mathrm{C}$ & $\mathrm{P}$ & 1 & $*$ & $*$ & $*$ & $*$ & $*$ & $*$ \\
\hline
\end{tabular}




\begin{tabular}{|c|c|c|c|c|c|c|c|c|c|c|}
\hline \multirow[b]{2}{*}{ Espèces et auteurs } & \multirow{2}{*}{$\begin{array}{l}\mathrm{F} \\
\mathrm{B}\end{array}$} & \multirow{2}{*}{$\begin{array}{c}\text { Sit } \\
\mathrm{e}\end{array}$} & \multirow[b]{2}{*}{$\mathrm{Nu}$} & \multirow{2}{*}{$\begin{array}{c}\mathrm{TB} \\
(\% \\
)\end{array}$} & \multicolumn{6}{|c|}{ Mois de floraison } \\
\hline & & & & & A & \begin{tabular}{|l|}
$M$ \\
$\mathrm{i}$
\end{tabular} & $\mathrm{J}$ & $\mathrm{Ji}$ & $\begin{array}{l}\mathrm{A} \\
\mathrm{O}\end{array}$ & $S$ \\
\hline \multicolumn{11}{|l|}{ Leguminosae-Papilionoideae } \\
\hline $\begin{array}{c}\text { Adenodolichos paniculatus (Hua) } \\
\text { Hutch. } \\
\text { \& Dalziel } \\
\end{array}$ & $\mathrm{a}$ & $\mathrm{C}$ & $\mathrm{N}$ & 3 & & & & & & $*$ \\
\hline Crotalaria retusa $\mathrm{L}$. & $\mathrm{H}$ & $\mathrm{K}$ & $\mathrm{N}$ & 1 & & & - & - & - & - \\
\hline $\begin{array}{l}\text { Desmodium gangeticum (L.) DC. } \\
\text { Var. } \\
\text { maculatum }(\mathrm{L}) \text { Baker } \\
\end{array}$ & $\mathrm{H}$ & $\begin{array}{l}\mathrm{K} / \\
\mathrm{T}\end{array}$ & $\mathrm{N} / \mathrm{N}$ & $1 / 1$ & & $\circ$ & $\bar{c}^{-}$ & & & \\
\hline Glycine $\max$ (L.) Merr. & $\mathrm{H}$ & $\mathrm{K}$ & $\mathrm{N}$ & 1 & & & & & - & - \\
\hline Indigofera dendroides Jacq. & $\mathrm{H}$ & $\begin{array}{l}\mathrm{K} / \\
\mathrm{T}\end{array}$ & $\mathrm{N} / \mathrm{N}$ & $1 / 1$ & & & & & & $\bar{\circ}^{\circ}$ \\
\hline Indigofera garckeana Vatke & $\mathrm{a}$ & $\mathrm{T}$ & $\mathrm{N}$ & 1 & & & & & $\circ$ & $\circ$ \\
\hline Indigofera leprieurii Baker $\mathrm{f}$. & $\mathrm{H}$ & $\mathrm{T}$ & $\mathrm{N}$ & 1 & & & & & & $\circ$ \\
\hline $\begin{array}{c}\text { Millettia thonningii (Schumach. } \\
\text { \&Thonn.) } \\
\text { Baker }\end{array}$ & A & $\mathrm{K}$ & $\mathrm{N}$ & 3 & - & & & & & \\
\hline $\begin{array}{c}\text { Philenoptera cyanescens (Schumach. } \\
\& \\
\text { Thonn.) Roberty } \\
\end{array}$ & $\mathrm{a}$ & $\mathrm{K}$ & $\mathrm{N}$ & 4 & & & & & - & \\
\hline Tephrosia flexuosa G.Don & $\mathrm{H}$ & $\mathrm{T}$ & $\mathrm{N}$ & 1 & & & & & & $\circ$ \\
\hline \multicolumn{11}{|l|}{ Loganiaceae } \\
\hline Strychnos spinosa Lam. & $\mathrm{a}$ & $\begin{array}{l}\mathrm{C} / \\
\mathrm{T}\end{array}$ & $\mathrm{N} / \mathrm{N}$ & 1 & & $\begin{array}{ll}* \\
\circ \\
\end{array}$ & & & & \\
\hline \multicolumn{11}{|l|}{ Malvaceae } \\
\hline $\begin{array}{l}\text { Abelmoschus esculentus (L.) } \\
\text { Moench. }\end{array}$ & $\mathrm{H}$ & $\mathrm{T}$ & NP & 2 & & & & & $\circ$ & $\circ$ \\
\hline Gossypium hirsutum L. & $\mathrm{H}$ & $\begin{array}{l}\mathrm{C} / \\
\mathrm{K} / \\
\mathrm{T}\end{array}$ & $\begin{array}{l}\mathrm{N} / \mathrm{N} \\
\mathrm{P} / \mathrm{NP}\end{array}$ & $\begin{array}{l}1 / 2 \\
/ 1\end{array}$ & & & - & - & - & $\begin{array}{l}* \\
- \\
\circ\end{array}$ \\
\hline Sida acuta Burm.f. ssp. acuta & $\mathrm{H}$ & $\mathrm{C}$ & NP & 2 & & & $*$ & $*$ & $*$ & $*$ \\
\hline $\begin{array}{c}\text { Sida acuta Burm.f. ssp. carpinifolia } \\
\text { (L.f.) } \\
\text { Borss. Waalk. }\end{array}$ & $\mathrm{H}$ & $\mathrm{C}$ & NP & 2 & & & & & & $*$ \\
\hline Sida garckeana Pol. & $\mathrm{H}$ & $\mathrm{K}$ & $\mathrm{P}$ & 1 & & & & & & - \\
\hline \multicolumn{11}{|l|}{ Moraceae } \\
\hline Ficus sycomorus L. & A & $\mathrm{C}$ & $\mathrm{J}$ & 10 & $\mathrm{fr}$ & & & & & \\
\hline \multicolumn{11}{|l|}{ Moringaceae } \\
\hline Moringa oleifera Lam. & $\mathrm{a}$ & $\mathrm{K}$ & $\mathrm{NP}$ & 1 & & & - & - & & \\
\hline \multicolumn{11}{|l|}{ Musaceae } \\
\hline Musa acuminata $\mathrm{L}$. & $\mathrm{H}$ & $\mathrm{K}$ & $\mathrm{N}$ & 3 & & & & & - & - \\
\hline
\end{tabular}




\begin{tabular}{|c|c|c|c|c|c|c|c|c|c|c|}
\hline \multirow[b]{2}{*}{ Espèces et auteurs } & \multirow{2}{*}{$\begin{array}{l}\mathrm{F} \\
\mathrm{B}\end{array}$} & \multirow{2}{*}{$\begin{array}{c}\text { Sit } \\
\mathrm{e}\end{array}$} & \multirow[b]{2}{*}{$\mathrm{Nu}$} & \multirow{2}{*}{$\begin{array}{l}\mathrm{TB} \\
(\% \\
) \\
\end{array}$} & \multicolumn{6}{|c|}{ Mois de floraison } \\
\hline & & & & & A & $\begin{array}{c}\mathrm{M} \\
\mathrm{i}\end{array}$ & $\mathbf{J}$ & $\mathrm{Ji}$ & $\begin{array}{c}\mathrm{A} \\
\mathrm{O}\end{array}$ & $\mathrm{S}$ \\
\hline \multicolumn{11}{|l|}{ Myrtaceae } \\
\hline $\begin{array}{l}\text { Syzygium guineense (Willd.) DC. } \\
\text { var. guineense }\end{array}$ & $\mathrm{a}$ & $\mathrm{K}$ & NP & 2 & & & & - & & \\
\hline $\begin{array}{l}\text { Syzygium guineense (Willd.) DC. } \\
\text { var. macrocarpum (Engl.) F.White }\end{array}$ & $\mathrm{a}$ & $\mathrm{K}$ & NP & 2 & & & & - & & \\
\hline \multicolumn{11}{|l|}{ Nyctaginaceae } \\
\hline Boerhavia diffusa $\mathrm{L}$. & $\mathrm{H}$ & $\mathrm{T}$ & NP & 2 & & & $\circ$ & $\circ$ & $\circ$ & \\
\hline \multicolumn{11}{|l|}{ Olacaceae } \\
\hline Ximenia americana $\mathrm{L}$. & $\mathrm{a}$ & $\mathrm{T}$ & $\mathrm{N}$ & 5 & $\circ$ & & & & & \\
\hline \multicolumn{11}{|l|}{ Pedaliaceae } \\
\hline Ceratotheca sesamoides Endl. & $\mathrm{H}$ & $\mathrm{K}$ & $\mathrm{N}$ & 1 & & & - & - & - & - \\
\hline \multicolumn{11}{|l|}{ Poaceae } \\
\hline $\begin{array}{l}\text { Hyparrhenia involucrata Stapf } \\
\text { var.involucrata }\end{array}$ & $\mathrm{H}$ & $\mathrm{T}$ & $\mathrm{P}$ & 1 & & & & & & $\circ$ \\
\hline $\begin{array}{c}\text { Hyperthelia dissoluta (Nees ex } \\
\text { Steud.) } \\
\text { W.D.Clayton }\end{array}$ & $\mathrm{H}$ & $\mathrm{T}$ & $\mathrm{P}$ & 1 & & & & & $\circ$ & $\circ$ \\
\hline Sporobolus pyramidalis P.Beauv. & $\mathrm{H}$ & $\begin{array}{c}\mathrm{C} / \\
\mathrm{T}\end{array}$ & $\mathrm{P} / \mathrm{P}$ & $1 / 2$ & & & & & $\begin{array}{l}* \\
\end{array}$ & $*$ \\
\hline Zea mays $\mathrm{L}$. & $\mathrm{H}$ & $\begin{array}{l}\mathrm{C} / \\
\mathrm{K}\end{array}$ & $\mathrm{P} / \mathrm{P}$ & $2 / 1$ & & & & - & $\begin{array}{ll}* \\
- \\
\end{array}$ & $\begin{array}{ll}* \\
- \\
\end{array}$ \\
\hline \multicolumn{11}{|l|}{ Polygonaceae } \\
\hline Securidaca longepedunculata Fresen. & $\mathrm{a}$ & $\begin{array}{l}\mathrm{K} / \\
\mathrm{T} \\
\end{array}$ & $\mathrm{N} / \mathrm{N}$ & $1 / 2$ & $\begin{array}{l}- \\
\end{array}$ & $\circ$ & & $\circ$ & & \\
\hline \multicolumn{11}{|l|}{ Rhamnaceae } \\
\hline Ziziphus abyssinica A. Rich. & $\mathrm{a}$ & $\mathrm{T}$ & $\mathrm{N}$ & 6 & & & & $\circ$ & $\circ$ & $\circ$ \\
\hline Ziziphus mucronata Willd. & $\mathrm{a}$ & $\begin{array}{l}\mathrm{C} / \\
\mathrm{K}\end{array}$ & $\begin{array}{c}\mathrm{N} / \mathrm{N} \\
\mathrm{P}\end{array}$ & $3 / 5$ & & & & & - & $\begin{array}{ll}* \\
- \\
\end{array}$ \\
\hline \multicolumn{11}{|l|}{ Rubiaceae } \\
\hline Fadogia agrestis Schweinf. ex Hiern & $\mathrm{a}$ & $\begin{array}{l}\mathrm{C} / \\
\mathrm{K}\end{array}$ & $\mathrm{N} / \mathrm{N}$ & $3 / 1$ & & $\begin{array}{ll}* \\
- \\
\end{array}$ & $\begin{array}{l}* \\
- \\
\end{array}$ & $\begin{array}{ll}* \\
- \\
\end{array}$ & & \\
\hline $\begin{array}{c}\text { Feretia apodanthera Delile ssp. } \\
\text { apodanthera }\end{array}$ & $\mathrm{a}$ & $\mathrm{C}$ & $\mathrm{N}$ & 10 & $*$ & & & & & \\
\hline Mitracarpus hirtus (L.) DC. & $\mathrm{H}$ & $\begin{array}{c}\mathrm{C} / \\
\mathrm{T}\end{array}$ & $\mathrm{N} / \mathrm{N}$ & $1 / 1$ & & & & & $*$ & $*$ \\
\hline Mitragyna inermis (Willd.) Kuntze & A & $\begin{array}{l}\mathrm{C} / \\
\mathrm{K}\end{array}$ & $\mathrm{N} / \mathrm{N}$ & 3 & & & & & $\begin{array}{l}* \\
\\
- \\
\circ\end{array}$ & $\begin{array}{l}* \\
\circ\end{array}$ \\
\hline $\begin{array}{l}\text { Sarcocephalus latifolius (Sm.) } \\
\text { E.A.Bruce }\end{array}$ & $\mathrm{a}$ & $\begin{array}{l}\mathrm{C} / \\
\mathrm{T}\end{array}$ & $\mathrm{N} / \mathrm{N}$ & $2 / 1$ & $*$ & $\begin{array}{l}* \\
* \\
\circ\end{array}$ & $\circ$ & $*$ & & \\
\hline $\begin{array}{l}\text { Crossopteryx febrifuga (G.Don) } \\
\text { Benth. }\end{array}$ & $\mathrm{a}$ & $\begin{array}{l}\mathrm{C} / \\
\mathrm{T}\end{array}$ & $\begin{array}{c}\mathrm{N} / \mathrm{N} \\
\mathrm{P} \\
\end{array}$ & $1 / 2$ & $\begin{array}{l}* \\
* \\
\circ\end{array}$ & $\begin{array}{l}* \\
\circ \\
\end{array}$ & $\begin{array}{l}* \\
\circ \\
\end{array}$ & $\circ$ & & \\
\hline
\end{tabular}




\begin{tabular}{|c|c|c|c|c|c|c|c|c|c|c|}
\hline \multirow[b]{2}{*}{ Espèces et auteurs } & \multirow{2}{*}{$\begin{array}{l}\mathrm{F} \\
\mathrm{B}\end{array}$} & \multirow[b]{2}{*}{$\begin{array}{c}\text { Sit } \\
\mathrm{e}\end{array}$} & \multirow[b]{2}{*}{$\mathrm{Nu}$} & \multirow{2}{*}{$\begin{array}{c}\mathrm{TB} \\
(\% \\
)\end{array}$} & \multicolumn{6}{|c|}{ Mois de floraison } \\
\hline & & & & & A & $\mathrm{M}$ & $\mathrm{J}$ & $\mathrm{Ji}$ & $\begin{array}{c}\mathrm{A} \\
\mathrm{O}\end{array}$ & $S$ \\
\hline Gardenia erubescens Stapf \& Hutch. & $\mathrm{a}$ & $\begin{array}{c}\mathrm{C} / \\
\mathrm{K} / \\
\mathrm{T}\end{array}$ & $\begin{array}{c}\mathrm{NP} / \\
\mathrm{N} / \mathrm{N} \\
\mathrm{P}\end{array}$ & $\begin{array}{l}2 / 2 \\
/ 1\end{array}$ & $*$ & $*$ & $\circ$ & $*$ & & \\
\hline $\begin{array}{l}\text { Spermacoce stachydea DC. Var. } \\
\text { stachydea }\end{array}$ & $\mathrm{H}$ & $\begin{array}{l}\mathrm{C} / \\
\mathrm{K}\end{array}$ & $\begin{array}{l}\mathrm{NP} / \\
\mathrm{NP}\end{array}$ & $1 / 2$ & & & & & - & * \\
\hline \multicolumn{11}{|l|}{ Sapindaceae } \\
\hline Allophylus africanus P. Beauv. & $\mathrm{a}$ & $\mathrm{K}$ & $\mathrm{N}$ & 4 & & & & & - & \\
\hline Paullinia pinnata $\mathrm{L}$. & $\mathrm{L}$ & K & $\mathrm{N}$ & 5 & & - & - & - & & \\
\hline \multicolumn{11}{|l|}{ Sapotaceae } \\
\hline $\begin{array}{l}\text { Vitellaria paradoxa C.F. Gaertn. Ssp. } \\
\text { Paradoxa }\end{array}$ & A & $\begin{array}{l}\mathrm{C} / \\
\mathrm{K}\end{array}$ & $\begin{array}{l}\mathrm{NPR} \\
/ \mathrm{NP} \\
\mathrm{R}\end{array}$ & $\begin{array}{c}20 / \\
15\end{array}$ & * & & & & & \\
\hline \multicolumn{11}{|l|}{ Scrophulariaceae } \\
\hline Striga hermonthica (Delile) Benth. & $\mathrm{P}$ & $\mathrm{C}$ & $\mathrm{N}$ & 1 & & & & & * & $*$ \\
\hline \multicolumn{11}{|l|}{ Sterculiaceae } \\
\hline Sterculia setigera Delile & A & $\begin{array}{l}\mathrm{C} / \\
\mathrm{K} / \\
\mathrm{T}\end{array}$ & $\begin{array}{c}\mathrm{N} / \mathrm{N} / \\
\mathrm{N}\end{array}$ & $\begin{array}{l}2 / 1 \\
13\end{array}$ & $\begin{array}{ll}* \\
\\
\end{array}$ & $\begin{array}{c}* \\
\circ\end{array}$ & & & & \\
\hline \multicolumn{11}{|l|}{ Tiliaceae } \\
\hline Grewia bicolor Juss. & $\mathrm{a}$ & $\begin{array}{l}\mathrm{C} / \\
\mathrm{K}\end{array}$ & $\mathrm{N} / \mathrm{N}$ & $3 / 5$ & $\begin{array}{ll}* \\
- \\
\end{array}$ & $\begin{array}{ll}* \\
- \\
\end{array}$ & $*$ & $*$ & $*$ & $*$ \\
\hline Grewia lasiodiscus K.Schum. & $\mathrm{a}$ & $\begin{array}{c}\mathrm{K} / \\
\mathrm{T}\end{array}$ & $\mathrm{N} / \mathrm{N}$ & $1 / 1$ & $\bar{\circ}$ & - & $\bar{\circ}$ & - & & \\
\hline Grewia cissoides Hutch. \& Dalzeil & $\mathrm{a}$ & $\mathrm{K}$ & NP & 2 & & - & & & & \\
\hline Grewia mollis Juss. & $\mathrm{a}$ & $\mathrm{T}$ & $\begin{array}{l}\mathrm{NP} / \\
\mathrm{NP}\end{array}$ & $1 / 1$ & $\circ$ & $\circ$ & $\circ$ & $\circ$ & & \\
\hline \multicolumn{11}{|l|}{ Verbenaceae } \\
\hline Gmelina arborea Roxb. & A & $\mathrm{K}$ & $\mathrm{N}$ & 4 & - & & & & & \\
\hline Lantana ukambensis (Vatke) Verdc. & $\mathrm{H}$ & $\mathrm{T}$ & $\mathrm{N}$ & 1 & & & & & $\circ$ & \\
\hline Stachytarpheta indica (L.) Vahl & $\mathrm{H}$ & $\mathrm{C}$ & $\mathrm{N}$ & 2 & $*$ & & & & & $*$ \\
\hline Vitex simplicifolia Oliv. & $\mathrm{a}$ & $\begin{array}{c}\mathrm{K} / \\
\mathrm{T}\end{array}$ & $\mathrm{N} / \mathrm{N}$ & $1 / 1$ & $\circ$ & $-\bar{c}$ & & & & \\
\hline Tectona grandis L. f. & A & $\mathrm{K}$ & NP & 3 & & & & - & - & \\
\hline \multicolumn{11}{|l|}{ Vitaceae } \\
\hline Cissus populnea Guill. \& Perr. & $\mathrm{L}$ & $\begin{array}{l}\mathrm{C} / \\
\mathrm{K}\end{array}$ & $\mathrm{N} / \mathrm{N}$ & 1 & - & - & & $\begin{array}{ll}* \\
-\end{array}$ & $*$ & \\
\hline $\begin{array}{c}\text { Cyphostemma adenocaule (Steud.) } \\
\text { Desc }^{\text {Con }}\end{array}$ & $\mathrm{L}$ & $\mathrm{C}$ & $\mathrm{N}$ & 15 & & & & & $*$ & $*$ \\
\hline \multicolumn{11}{|l|}{ Zygophyllaceae } \\
\hline Balanites aegyptiaca (L.) Delile & A & $\begin{array}{l}\mathrm{C} / \\
\mathrm{T}\end{array}$ & $\begin{array}{c}\mathrm{NP} / \\
\mathrm{N}\end{array}$ & $4 / 1$ & & & $\circ$ & $\begin{array}{l}* \\
*\end{array}$ & $*$ & \\
\hline
\end{tabular}




\section{Spectre pollinique des miels analysés}

L'analyse pollinique des 34 échantillons de miel a permis d'identifier au total 73 taxons polliniques appartenant à 46 genres et 38 familles. Parmi ces taxons polliniques, 43 sont identifiés jusqu'au niveau espèce (soit 58,9\% du total), 06 au niveau genre (soit 8,2\%) et 24 au niveau famille (soit $32,8 \%$ ). La richesse pollinique totale des échantillons de miel analysés est de 10469 pollens. Elle varie de 0 à 4346 grains de pollens par échantillon. Le tableau 3 montre que la famille des Combretaceae est la famille la plus riche en pollens avec un pourcentage de $52 \%$, suivie de celle des Leguminosae avec un taux de 16,5\% et enfin celle des Anacardiaceae avec un taux 8,4\%.

En outre, le tableau 3 souligne que le mois de septembre est celui durant lequel on note la forte récolte de grains de pollen par les butineuses avec un total de 5097 pollens, suivi du mois d'avril avec 3654 pollens. Au cours du mois d'août aucun échantillon de miel n'a pu être récolté.

Tableau 3: Spectre pollinique des échantillons de miels de la saison humide

\begin{tabular}{|c|c|c|c|c|c|c|c|c|}
\hline Taxons polliniques & $\mathrm{Av}$ & Mi & Jn & $\mathrm{Ju}$ & $\begin{array}{l}\mathrm{A} \\
\mathrm{O}\end{array}$ & Sept & Total & $\begin{array}{c}\text { Densité relative } \\
(\%)\end{array}$ \\
\hline \multicolumn{9}{|l|}{ Acanthaceae } \\
\hline Acanthaceae undiff. & 0 & 0 & 0 & 0 & 0 & 5 & 5 & 0,05 \\
\hline \multicolumn{9}{|l|}{ Amaranthaceae } \\
\hline Amaranthaceae undiff. & 0 & 0 & 0 & 1 & 0 & 0 & 1 & 0,01 \\
\hline Gomphrena celosoides & 0 & 0 & 0 & 0 & 0 & 95 & 95 & 0,91 \\
\hline \multicolumn{9}{|l|}{ Anacardiaceae } \\
\hline Anarcadiaceae undiff. & 50 & $\begin{array}{c}20 \\
8 \\
\end{array}$ & 0 & 15 & 0 & 15 & 288 & 2,75 \\
\hline Ozoroa pulcherrima & 539 & 46 & 0 & 0 & 0 & 0 & 585 & 5,59 \\
\hline \multicolumn{9}{|l|}{ Annonaceae } \\
\hline Annona senegalensis & 0 & 0 & 0 & 7 & 0 & 0 & 7 & 0,07 \\
\hline \multicolumn{9}{|l|}{ Apocynaceae } \\
\hline Apocynaceae undiff. & 6 & $\begin{array}{c}11 \\
2 \\
\end{array}$ & 0 & 5 & 0 & 0 & 123 & 1,17 \\
\hline \multicolumn{9}{|l|}{ Arecaceae } \\
\hline Arecaceae undiff. & 2 & 0 & 3 & 0 & 0 & 0 & 5 & 0,05 \\
\hline Eleais guineensis & 43 & 5 & 5 & 1 & 0 & 0 & 54 & 0,52 \\
\hline \multicolumn{9}{|l|}{ Asclepiadaceae } \\
\hline Asclepiadaceae undiff. & 2 & 0 & 0 & 0 & 0 & 0 & 2 & 0,02 \\
\hline \multicolumn{9}{|l|}{ Asteraceae } \\
\hline Asteraceae undiff. & 0 & 0 & 0 & 0 & 0 & 2 & 2 & 0,02 \\
\hline Capparaceae & & & & & & & & \\
\hline
\end{tabular}




\begin{tabular}{|c|c|c|c|c|c|c|c|c|}
\hline Taxons polliniques & $\mathrm{Av}$ & $\mathrm{Mi}$ & Jn & $\mathrm{Ju}$ & $\begin{array}{l}\mathrm{A} \\
\mathrm{O}\end{array}$ & Sept & Total & $\begin{array}{c}\text { Densité relative } \\
(\%)\end{array}$ \\
\hline Cleome viscosa & 0 & 3 & 0 & 0 & 0 & 0 & 3 & 0,03 \\
\hline \multicolumn{9}{|l|}{ Celastraceae } \\
\hline Celastraceae undiff. & 2 & 0 & 0 & 0 & 0 & 0 & 2 & 0,02 \\
\hline \multicolumn{9}{|l|}{ Combretaceae } \\
\hline Combretaceae undiff. & 211 & 89 & 0 & 4 & 0 & 0 & 304 & 2,90 \\
\hline Combretum collinum & $\begin{array}{c}108 \\
3 \\
\end{array}$ & 0 & 0 & 0 & 0 & $\begin{array}{c}401 \\
4 \\
\end{array}$ & 5097 & 48,69 \\
\hline Combretum-type & 2 & 0 & 0 & 0 & 0 & 0 & 2 & 0,02 \\
\hline Terminalia-type & 0 & 44 & 0 & 3 & 0 & 0 & 47 & 0,45 \\
\hline \multicolumn{9}{|l|}{ Commelinaceae } \\
\hline Commelina-type & 0 & 0 & 0 & 0 & 0 & 69 & 69 & 0,66 \\
\hline Commelinaceae & 4 & 6 & 0 & 2 & 0 & 4 & 16 & 0,15 \\
\hline Cyanotis lanata & 4 & 0 & 0 & 0 & 0 & 143 & 147 & 1,40 \\
\hline \multicolumn{9}{|l|}{ Connaraceae } \\
\hline Rourea coccinea & 0 & 11 & 0 & 0 & 0 & 0 & 11 & 0,11 \\
\hline \multicolumn{9}{|l|}{ Convolvulaceae } \\
\hline Convolvulaceae undiff. & 2 & 0 & 0 & 0 & 0 & 222 & 224 & 2,14 \\
\hline \multicolumn{9}{|l|}{ Cyperaceae } \\
\hline Cyperaceae & 0 & 0 & 0 & 1 & 0 & 1 & 2 & 0,02 \\
\hline \multicolumn{9}{|l|}{ Ebenaceae } \\
\hline Diospyros mespiliformis & 28 & 17 & 5 & 11 & 0 & 0 & 61 & 0,58 \\
\hline Ebenaceae undiff. & 1 & 0 & 0 & 0 & 0 & 0 & 1 & 0,01 \\
\hline \multicolumn{9}{|l|}{ Euphorbiaceae } \\
\hline Bridelia ferruginea & 13 & 3 & 0 & 0 & 0 & 6 & 22 & 0,21 \\
\hline Euphorbiaceae undiff. & 81 & 17 & $\begin{array}{l}1 \\
2 \\
\end{array}$ & $\begin{array}{c}12 \\
3 \\
\end{array}$ & 0 & 16 & 249 & 2,38 \\
\hline Flueggea virosa & 0 & 4 & 0 & 0 & 0 & 0 & 4 & 0,04 \\
\hline Hymenocardia acida & 0 & 1 & 0 & 0 & 0 & 0 & 1 & 0,01 \\
\hline Margaritaria discoidea & 448 & 0 & 0 & 0 & 0 & 0 & 448 & 4,28 \\
\hline \multicolumn{9}{|l|}{ Irvingiaceae } \\
\hline Irvingia gabonensis & 2 & 0 & 0 & 0 & 0 & 0 & 2 & 0,02 \\
\hline \multicolumn{9}{|l|}{ Lamiaceae } \\
\hline Basilicum polystachyon & 0 & 0 & 0 & 0 & 0 & 2 & 2 & 0,02 \\
\hline Becium obovatum & 1 & 0 & 0 & 0 & 0 & 0 & 1 & 0,01 \\
\hline $\begin{array}{c}\text { Leguminosae- } \\
\text { Caesalpinioideae }\end{array}$ & & & & & & & & \\
\hline
\end{tabular}




\begin{tabular}{|c|c|c|c|c|c|c|c|c|}
\hline Taxons polliniques & Av & $\mathrm{Mi}$ & $\mathrm{Jn}$ & $\mathrm{Ju}$ & $\begin{array}{l}\mathrm{A} \\
\mathrm{O}\end{array}$ & Sept & Total & $\begin{array}{c}\text { Densité relative } \\
(\%)\end{array}$ \\
\hline Caesalpinoideae undiff. & 74 & 36 & $\begin{array}{l}1 \\
0 \\
\end{array}$ & 29 & 0 & 10 & 159 & 1,52 \\
\hline Cassia sieberiana & 1 & 1 & 0 & 0 & 0 & 0 & 2 & 0,02 \\
\hline Piliostigma thonningii & 394 & 0 & 0 & 0 & 0 & 0 & 394 & 3,76 \\
\hline Tamarindus indica & 77 & 24 & 0 & 0 & 0 & 0 & 101 & 0,96 \\
\hline \multicolumn{9}{|l|}{ Leguminosae-Mimosoideae } \\
\hline Acacia dudgeonii & 26 & 0 & 0 & 0 & 0 & 0 & 26 & 0,25 \\
\hline Dichrostachys cinerea & 10 & 1 & 0 & 0 & 0 & 0 & 11 & 0,11 \\
\hline Entada africana & 16 & 0 & 0 & 0 & 0 & 0 & 16 & 0,15 \\
\hline Parkia biglobosa & 120 & 76 & 0 & $\begin{array}{c}52 \\
9 \\
\end{array}$ & 0 & 0 & 725 & 6,93 \\
\hline \multicolumn{9}{|l|}{ Leguminosae-Papilionoideae } \\
\hline Crotalaria retusa & 5 & 0 & 0 & 0 & 0 & 0 & 5 & 0,05 \\
\hline Lonchocarpus laxiflorus & 0 & 0 & 0 & 3 & 0 & 0 & 3 & 0,03 \\
\hline Papilionoideae & 194 & 17 & 0 & 78 & 0 & 3 & 292 & 2,79 \\
\hline Tephrosia purpurea & 0 & 0 & 0 & 0 & 0 & 1 & 1 & 0,01 \\
\hline \multicolumn{9}{|l|}{ Loganiaceae } \\
\hline Loganiaceae undiff. & 6 & 0 & 0 & 0 & 0 & 0 & 6 & 0,06 \\
\hline Strychnos spinosa & 1 & 0 & 0 & 0 & 0 & 0 & 1 & 0,01 \\
\hline \multicolumn{9}{|l|}{ Loranthaceae } \\
\hline Tapinanthus dodonerfolius & 5 & 0 & 0 & 0 & 0 & 0 & 5 & 0,05 \\
\hline Tapinanthus-type & 0 & 0 & 0 & 6 & 0 & 0 & 6 & 0,06 \\
\hline \multicolumn{9}{|l|}{ Malvaceae } \\
\hline Malvaceae undiff. & 0 & 0 & 1 & 2 & 0 & 47 & 50 & 0,48 \\
\hline Sida acuta & 0 & 0 & 0 & 0 & 0 & 8 & 8 & 0,08 \\
\hline \multicolumn{9}{|l|}{ Meliaceae } \\
\hline Meliaceae undiff. & 0 & 0 & 0 & 6 & 0 & 0 & 6 & 0,06 \\
\hline \multicolumn{9}{|l|}{ Moraceae } \\
\hline Ficus-type & 6 & 0 & 0 & 0 & 0 & 0 & 6 & 0,06 \\
\hline \multicolumn{9}{|l|}{ Myrtaceae } \\
\hline Syzygium guineense & 0 & 1 & 0 & 0 & 0 & 67 & 68 & 0,65 \\
\hline \multicolumn{9}{|l|}{ Nyctaginaceae } \\
\hline Boerhavia diffusa & 7 & 0 & 0 & 0 & 0 & 0 & 7 & 0,07 \\
\hline \multicolumn{9}{|l|}{ Poaceae } \\
\hline Poaceae undiff. & 27 & 1 & 3 & 12 & 0 & 145 & 188 & 1,80 \\
\hline
\end{tabular}




\begin{tabular}{|c|c|c|c|c|c|c|c|c|}
\hline Taxons polliniques & Av & $\mathrm{Mi}$ & $\mathrm{Jn}$ & $\mathrm{Ju}$ & $\begin{array}{c}\mathrm{A} \\
\mathrm{O}\end{array}$ & Sept & Total & $\begin{array}{c}\text { Densité relative } \\
(\%)\end{array}$ \\
\hline \multicolumn{9}{|l|}{ Rhamnaceae } \\
\hline Ziziphus abyssinica & 0 & 0 & 2 & 2 & 0 & 0 & 4 & 0,04 \\
\hline Ziziphus mucronata & 0 & 0 & 0 & 0 & 0 & 7 & 7 & 0,07 \\
\hline \multicolumn{9}{|l|}{ Rubiaceae } \\
\hline Crossopterix febrifuga & 16 & 0 & 0 & 0 & 0 & 0 & 16 & 0,15 \\
\hline Rubiaceae undiff. & 16 & 11 & 6 & 1 & 0 & 167 & 201 & 1,92 \\
\hline Sarcocephalus latifolius & 0 & 0 & 1 & 0 & 0 & 0 & 1 & 0,01 \\
\hline \multicolumn{9}{|l|}{ Sapindaceae } \\
\hline Paullinia pinnata & 17 & 0 & 0 & 0 & 0 & 0 & 17 & 0,16 \\
\hline Sapindaceae undiff. & 0 & 0 & 0 & 1 & 0 & 0 & 1 & 0,01 \\
\hline \multicolumn{9}{|l|}{ Sapotaceae } \\
\hline Sapotaceae undiff. & 0 & 0 & 0 & 0 & 0 & 4 & 4 & 0,04 \\
\hline Trichilia emetica & 10 & 0 & 0 & 0 & 0 & 0 & 10 & 0,10 \\
\hline Vitellaria paradoxa & 0 & 0 & 0 & 87 & 0 & 0 & 87 & 0,83 \\
\hline \multicolumn{9}{|l|}{ Sterculiaceae } \\
\hline Sterculia setigera & 1 & 0 & 0 & 0 & 0 & 0 & 1 & 0,01 \\
\hline Waltheria indica & 1 & 0 & 0 & 0 & 0 & 0 & 1 & 0,01 \\
\hline \multicolumn{9}{|l|}{ Verbebaceae } \\
\hline Stachytarpheta indica & 41 & 0 & 0 & 0 & 0 & 0 & 41 & 0,39 \\
\hline \multicolumn{9}{|l|}{ Vitaceae } \\
\hline Cissus populnea & 7 & 0 & 0 & 0 & 0 & 0 & 7 & 0,07 \\
\hline Vitaceae undiff. & 0 & 2 & 0 & 0 & 0 & 0 & 2 & 0,02 \\
\hline Leea guineensis & 0 & 0 & 0 & 0 & 0 & 44 & 44 & 0,42 \\
\hline \multicolumn{9}{|l|}{ Zingiberaceae } \\
\hline Siphonochilus aethiopicus & 1 & 0 & 0 & 0 & 0 & 0 & 1 & 0,01 \\
\hline Indéterminé & 23 & 0 & 0 & 5 & 0 & 0 & 28 & 0,27 \\
\hline Total & $\begin{array}{c}365 \\
4 \\
\end{array}$ & $\begin{array}{c}73 \\
6 \\
\end{array}$ & $\begin{array}{l}4 \\
8 \\
\end{array}$ & $\begin{array}{c}93 \\
4 \\
\end{array}$ & 0 & $\begin{array}{c}509 \\
7 \\
\end{array}$ & $\begin{array}{c}1046 \\
9 \\
\end{array}$ & 100 \\
\hline
\end{tabular}

\section{Densité relative}

Quatre classes de pollen (taxon) sont distinguées suivant la valeur des densités obtenues. La densité relative (Dr) de pollens par taxons identifiés varie d'un échantillon à un autre. Ce qui signifie qu'un pollen dominant dans un échantillon peut ne plus l'être dans un autre et peut devenir soit un pollen d'accompagnement, soit isolé important ou encore isolé. C'est le cas des pollens Parkia biglobosa (Mimosaceae), pollens isolés importants à Cobly 
mais pollens d'accompagnement à Kouandé. Les différents taxons sont repartis selon leurs densités dans le Tableau 4.

Tableau 4: Répartition des richesses taxonomiques en fonction des classes de dominance

\begin{tabular}{|c|c|c|c|}
\hline \multirow{2}{*}{ Classe de densité } & \multicolumn{3}{|c|}{ Richesse taxonomique } \\
\hline & Cobly & Kouandé & Tanguiéta \\
\hline $\begin{array}{l}\text { Pollens dominants } \\
\text { Dr } \geq 45 \%\end{array}$ & Néant & Néant & $\begin{array}{l}\text { Combretum } \\
\text { collinum }\end{array}$ \\
\hline $\begin{array}{c}\text { Pollens } \\
\text { d'accompagnements } \\
\text { ou importants } \\
16 \leq \mathrm{Dr} \leq 45 \%\end{array}$ & $\begin{array}{c}\text { Ozoroa } \\
\text { pulcherima, } \\
\text { Margaritaria } \\
\text { discoidea; }\end{array}$ & Parkia biglobosa; & Néant \\
\hline $\begin{array}{l}\text { Pollens isolés } \\
\text { importants } \\
3 \% \leq \mathrm{Dr} \leq 16 \%\end{array}$ & $\begin{array}{l}\text { Tamarindus } \\
\text { indica, Parkia } \\
\text { biglobosa; }\end{array}$ & Combretum type; & $\begin{array}{l}\text { Ipomoea type, } \\
\text { Piliostigma } \\
\text { thonningii. }\end{array}$ \\
\hline $\begin{array}{c}\text { Pollens isolés } \\
\text { Dr } \leq 3 \%\end{array}$ & $\begin{array}{l}\text { Becium obovatum, } \\
\text { Dichrostachys } \\
\text { cinerea, Strychnos } \\
\text { spinosa, Entada } \\
\text { africana, Trichilia } \\
\text { emetica, Bridelia } \\
\text { ferruginea. }\end{array}$ & $\begin{array}{c}\text { Hymenocardia acida, } \\
\text { Siphonochilus } \\
\text { aethiopicus, } \\
\text { Sterculia setigera, } \\
\text { Syzygium guineense, } \\
\text { Cleome viscosa, } \\
\text { Sida acuta. }\end{array}$ & $\begin{array}{c}\text { Tephrosia } \\
\text { purpurea, } \\
\text { Basilicum } \\
\text { polystachyon, } \\
\text { Crossopterix } \\
\text { febrifuga, Paulinia } \\
\text { pinnata, } \\
\text { Acacia dudgeonii, } \\
\text { Gomphrena } \\
\text { celosoides. }\end{array}$ \\
\hline
\end{tabular}

La richesse taxonomique des échantillons de miels analysés varie d'un rucher à un autre : 16 pour Cobly, 49 pour Kouandé et 35 pour Tanguiéta. Les échantillons de miels provenant des ruchers de Kouandé et de Tanguiéta sont respectivement trois fois et deux fois plus diversifiés en taxons polliniques que ceux venant du rucher de Cobly.

\section{Contribution spécifique de l'analyse pollinique des miels et de l'observation directe du terrain des plantes mellifères}

Les deux méthodes couplées portent la flore mellifère de la zone Ouest soudanienne à 129 espèces. L'observation directe de terrain a permis de recenser 79 espèces, soit $61,24 \%$ de la flore mellifère de la saison des pluies tandis que l'analyse pollinique en a uniquement contribué à hauteur de 20 espèces, soit 15,50\%. Toutefois, 30 espèces (soit 23,26\%) ont été à la fois identifiées sur le terrain et dans les miels analysés.

Parmi les espèces exclusivement identifiées dans les miels, 13 espèces étaient en fleurs au cours de la période d'étude mais n'ont pas été recensées comme plantes mellifères par la méthode d'observation directe. Il s'agit de Annona senegalensis, Basilicum polystachyon, Becium obovatum, Boerhavia diffusa, Elaeis guineensis, Ozoroa pulcherrima, Piliostigma thonningii, 
Rourea coccinea, Siphonochilus aethiopicus, Strychnos spinosa, Tapinanthus dodoneifolius, Tephrosia purpurea et waltheria indica. Cinq espèces, telles que Cassia sieberiana, Diospyros mespiliformis, Hymenocardia acida, Lonchocarpus laxiflorus et Trichilia emetica, ont été identifiées dans les miels alors qu'elles n'étaient pas en fleurs durant la saison des pluies. Aussi, Irvingia gabonensis et Leea guineensis sont-elles identifiées par l'analyse pollinique sans être rencontrées dans l'aire d'observation qui est de $1 \mathrm{~km}$ de rayon autour des ruchers.

Parmi les espèces nectarifères, 15 ont leurs pollens identifiés dans les miels. Deux d'entre elles présentent une densité relativement élevée à savoir Margaritaria discoidea (4,28\%) Combretum collinum (48,69\%).

\section{DISCUSSION}

La présente étude a permis d'identifier 129 espèces mellifères au cours de la saison humide. La richesse spécifique des miels analysés est de 43 taxons et les familles prédominantes sont les Combretaceae (52\%) et les Leguminosae (16,5\%). Cette richesse spécifique est nettement inférieure à celle de 109 espèces mellifères recensées par l'observation directe sur le terrain. Les familles importantes recencées sont les Leguminosae $(21,1 \%)$ et les Rubiaceae (7,3\%). La différence significative observée entre ces deux richesses spécifiques peut se justifier par le fait que les plantes exclusivement nectarifères représentent plus de $50 \%$ des plantes mellifères identifiées sur les trois sites du milieu d'étude. En considérant les deux méthodes d'étude des plantes mellifères, les familles des Leguminosae, des Combretaceae et des Rubiaceae sont prédominantes. Ceci n'est pas une particularité des formations végétales abritant les ruchers, mais une caractéristique générale de la flore de la zone soudanienne du Bénin (Akoègninou et al., 2006). L'observation directe du butinage et l'analyse pollinique des miels sont des méthodes d'étude complémentaires des plantes mellifères. Ceci confirme les résultats de Tossou et al. (2005 et 2011) dans la forêt classée de la Lama et dans l'arrondissement de Manigri.

La richesse spécifique totale de 129 espèces obtenue par cette étude est inférieure à celle de 225 espèces identifiées par les travaux d'analyse pollinique de Koudegnan (2015) en zone guinéenne au Togo. Par contre, elle reste supérieure aux 96 et 97 espèces identifiées par les travaux d'observation directe de Nombré (2003) respectivement dans les zones de Garango au Nordsoudanien et Nazinga au Sud-soudanien du Burkina-Faso et aussi aux 78 espèces trouvées par Nguemo et al. (2004) dans les miels de la zone soudanoguinéenne à l'Ouest du Cameroun. Cependant, elle est sensiblement similaire aux 121 taxons déterminés par Tossou et al. (2005) dans les miels de la forêt classée de la Lama au Sud du Bénin. Cette différence de diversité taxonomique peut être liée au nombre d'échantillons de miels analysés, au mode 
d'échantillonnage des miels analysés, à la période de récolte, à la méthode d'etude et à la diversité floristique des types de formation ayant servi de source d'affouragement aux abeilles (Nombré, 2003 ; Koudegnan 2012).

Le spectre pollinique de la zone d'étude montre que Combretum collinum est le taxon le plus abondant $(48,69 \%)$ dans les miels ; alors qu'il se révèle par l'observation directe sur le terrain comme une espèce exclusivement nectarifère et non intensément butinée. Ceci confirme le constat fait ci-dessus sur l'apport de la mellissopalynologie dans l'amélioration des connaissances sur la flore apicole. Cette forte densité relative des pollens de Combretum collinum serait due : par le fait que les abeilles butineuses récoltent très tôt les pollens de ladite espèce avant le passage de l'observateur; la période de disponibilité du pollen et du nectar serait différée durant la journée et aussi du fait que l'espèce constitue la seule source dominante de nutriments en période de disette (Nombré, 2003). De même la stratégie de recrutement des ouvrières pour une activité de butinage optimal de ce taxon pourrait justifier le poids important de ces pollens au début de la saison des pluies en zone soudanienne (Nombré et al., 2001).

La contribution de $15,50 \%$ de l'analyse pollinique est supérieure à celle de 11,90\% obtenue par Tossou et al. (2011) dans l'arrondissement de Manigri en zone soudano-guinéenne. Mais, elle reste nettement inférieure à celle de 36,40 notée dans la forêt classée de la Lama en zone guinéenne (Tossou et al., 2005). Parmi les 20 espèces identifiées uniquement par analyse des miels, 13 étaient en fleurs et n'avaient pas été recensés comme plantes mellifères par l'étude du comportement de butinage des abeilles. Cela est dû simplement au non simultanéité de l'activité de butinage des ouvrières sur ces différentes espèces et les moments d'observation directe sur le terrain. Les espèces telles que Cassia sieberiana, Diospyros mespiliformis, Hymenocardia acida, Lonchocarpus laxiflorus et Trichilia emetica fleurissent en seconde partie de la saison sèche (Arbonnier, 2009). Ainsi, la présence des pollens de ces taxons dans les miels analysés pourrait s'expliquer par le fait qu'ils constituent des réserves polliniques emmagasinées pendant leur floraison en saison sèche. En effet, selon Lobreau-Callen et Damblon (1994), les réserves de pollens ne sont entièrement épuisées de la ruche qu'après six mois de stockage alors qu'ici, la durée entre la période d'étude et la floraison desdites espèces est inférieure à six mois. Ce qui justifie leur présence dans les miels échantillonnés et analysés.

Au sein des espèces identifiées, Irvingia gabonensis et Leea guineensis sont les seules espèces situées hors des points d'échantillonnage ou de l'aire d'observation qui est de $1 \mathrm{~km}$ autour des ruchers. Ceci corrobore les constats faits par Tossou et al. (2005; 2011) et Demenou (2006), qui préconisent $1 \mathrm{~km}$ autour du rucher, contrairement à ceux de Nombré (2003) et Sawadogo (1993), qui recommandent respectivement $2 \mathrm{~km}$ et $3 \mathrm{~km}$ autour du rucher. 
Dans les espèces identifiées dans les miels, 15 sont reconnues exclusivement nectarifères par observation directe de terrain. Parmi celles-ci, 13 présentent une densité pollinique relative très faible. Leur présence dans les miels pourrait être expliquée par le fait que lors du butinage d'une espèce exclusivement nectarifère, les butineuses peuvent, par contamination retourner dans la ruche avec des pollens de ladite espèce (Yédomonhan, 2004 ; Tossou, 2011). Au sein de la même zone Ouest soudanienne, les plantes mellifères varient en fonction de la composition floristique de la végétation. Ceci a été déjà observé par Fohounfo (2002) et Yédomonhan (2004) qui ont travaillé respectivement à Kétou et dans la Lama en zone guinéenne et pourrait se justifier par la variation des facteurs climatiques dans ces zones qui aboutit à des subdivisions tels que les districts phytogéographiques selon Adomou (2005).

\section{Conclusion}

A l'issue de cette étude sur la connaissance et la diversité de la flore mellifère en zone Ouest soudanienne, 129 espèces ont été recensées dont 79 par observation directe sur le terrain, 20 par analyse pollinique et 30 identifiées à la fois par les deux méthodes. L'analyse pollinique des miels et l'observation directe de terrain sont donc deux méthodes d'étude qui se complètent pour l'amélioration des connaissances acquises sur la relation entre abeilles et plantes. La contribution de l'analyse pollinique à cette maîtrise de la flore apicole de la saison des pluies est de 15,50\%. Elle a permis aussi de montrer le statut mixte (nectarifère et pollinifère) de certaines espèces alors qu'elles étaient considérées comme exclusivement nectarifère par observation directe.

\section{Remerciement}

Nous remercions sincèrement le Ministère de l'Enseignement Supérieur et de la Recherche scientifique pour avoir soutenu financièrement ce travail.

\section{References:}

1. Adomou A.C. 2005. Vegetation patterns and environmental gradients in Benin: implications for biogeography and conservation. $\mathrm{PhD}$ thesis Wageningen University, Wageningen, 150 p.

2. Akoègninou A., van der Burg W. J. \& van der Maesen L. J. G. 2006. Flore analytique du Bénin. Backhuys Publishers, Wageningen, 1034 p.

3. Arbonnier M. 2009. Arbres, arbustes et lianes des zones sèches d'Afrique de l'Ouest. Edition Quae, MNHN, 573 p. 
4. Bonnefille R. \& Riollet G. 1980. Pollens des Savanes d'Afrique Orientale, $254 \mathrm{p}$.

5. Coulibaly S., Ouattara D., Edorh T., Koudégnan C. \& Kamanzi K. 2013. Diversité et configuration de la flore ligneuse autour d'un rucher en zone de transition forêt-savane de la Côte d'Ivoire. European Scientific Journal, 6(9) : 227-239.

6. De Layens G. \& Bonnier G. 1997. Cours complet d'apiculture et conduite d'un rucher isolé. Editions Belin, 458 p.

7. Dèmenou B. B. 2006. Inventaire des plantes mellifères et caractérisation pollinique des miels des élevages apicoles de la zone soudano-guinéenne de Manigri. Mémoire d'Ingénieur des Tavaux, Ecole Polytechnique d'Abomey-Calavi, Université d'Abomey-Calavi, Bénin, $68 \mathrm{p}$.

8. Erdtman G. 1969. Pollen and spores preparations: the acetolysis method. In handbook of palynology, Munksgaard, Copenhagen, 213216.

9. Faure P. 1977. Carte pédologique de reconnaissance de la République Populaire du Bénin à

10. 1/200 000 : feuille Djougou. Orstom, notice explicative 66(4), 49 p.

11. Feller-Demasly MJ \& Parent J. 1989.Analyse pollinique des miels de l'Ontario, Canada. Apidologie, 20 : 127-138.

12. Fohounfo H. T. 2002. Plantes mellifères et composition pollinique des miels de la petite saison des pluies et de la grande saison sèche au sud Bénin. Mémoire DIT, Université d'Abomey-Calavi, Bénin, 56 p.

13. Koudégnan C.M., Edorh M. T., Guelly K. A., Batawilla K. \& Akpagana K. 2012. Inventaire des taxons polliniques des miels de la Zone Guinéenne du Togo: cas des zones écofloristiques IV et V. European Scientific Journal, 26(8) : 37-50.

14. Koudégnan C.M. 2015. Contribution de la Palynologie à la caractérisation des miels et à la connaissance de la flore mellifères de la zone guinéenne du Togo.Thèse de Doctorat, Université de Lomé, Togo, $115 \mathrm{p}$.

15. Lobreau-Callen D., Darchen R. \& Le Thomas A. 1986. Apport de la palynologie à la connaissance des relations abeilles/plantes en savanes arborées du Togo et du Bénin. Apidologie, 17(4) : 279-306. 
16. Lobreau-Callen D. \& Damblon F. 1994. Spectre pollinique des miels de l'abeille Apis mellifera L. (Hymenoptera, Apidae) et zone de végétation en Afrique occidentale et méditerranéenne. Grana, 33 : 245-253.

17. Nguemo D. D., Foko J., Pinta J. Y., Ngouo L. V., Tchoumboue J. \& Zango P. 2004. Inventaire et identification des plantes mellifères de la zone soudano-guinéenne d'altitude de l'ouest Cameroun. Tropicultura, 22(3): 139-145.

18. Nguemo D. D., Tchoumboue J., Pinta J.Y. \& Zango P. 2008. Caractéristiques polliniques des plantes mellifères de la zone soudanoguinéenne d'altitude de l'ouest Cameroun. Tropicultura, 26(3) : 150154.

19. Nombré I., Sawadogo M. \& Guinko S. 2001. Etudes des plantes mellifères et de l'évolution du poids des ruches dans la zone de Garango (Province du Boulgou) centre-est du Burkina Faso. Bull. Techn. Apic., 28(4) : 176-180.

20. Nombré I. 2003. Etudes des potentialités mellifères de deux zones du Burkina Faso: Garango (province du Bouglou) et Nazinga (province du Nahouri). Thèse de Doctorat, Université de Ouagadougou, Burkina Faso, 156 p.

21. Sawadogo M. 1993. Contribution à l'étude du cycle des miellées et du cycle biologique annuel des colonies d'abeilles Apis mellifica adansonii Lat. à l'Ouest du Burkina Faso. Thèse de Doctorat, Université de Ouagadougou, Burkina Faso, 152 p.

22. Sowunmi M. A. 1973. Pollen grains of Nigerian plants. I Woody species. Grana, 13 : 145-186.

23. Sowunmi M. A. 1995. Pollen grains of Nigerian plants. II Woody species. Grana, 34 : 120-141.

24. Tossou G. M., Akoègninou A., Yédomonhan H., Batawila K. \& Akpagana K. 2005. Analyse pollinique des miels de la forêt classée de la Lama (Bénin) et son apport à la connaissance de la flore apicole. $J$. Rech. Sci. Univ. Lomé (Togo), série A, 7(1) : 83-92.

25. Tossou G. M., Yedomonhan H., Azokpota P., Akoegninou A., Doubogan P. \& Akpagana K. 2011. Analyse pollinique et caractérisation phytogéographique des miels vendus à Cotonou (Bénin). Cah Agric, 20 : 500-508. doi: 10.1684/agr.2011.052. 
26. Ybert J-P. 1979. Atlas de pollens de Côte d'Ivoire. Paris, Orstom, 40 p.

27. Yédomonhan H. 2004. Plantes mellifères et miels du Bénin : cas de la forêt classée de la Lama. Mémoire de DEA, Université de Lomé, Togo, $65 \mathrm{p}$.

28. Yédomonhan H. 2009a. Plantes mellifères et potentialités de production de miel en zones guinéenne et soudano-guinéenne au Bénin. Thèse de doctorat, Université d'Abomey-Calavi, Bénin, 294p.

29. Yédomonhan H., Tossou G.M., Akoègninou A., Demènou B.B. \& Traoré D. 2009b. Diversité des plantes mellifères de la zone soudanoguinéenne: cas de l'arrondissement de Manigri (Centre-Ouest du Bénin). Int. J. Biol. Chem. Sci., 3(2) : 355-366.

30. Yédomonhan H., Tossou G.M., Adomou C.A. \& Akoègninou A. 2009c. Flore mellifère du Bénin : cas de la forêt Classée de la Lama et de l'arrondissement de Manigri. Ann. Univ. Lomé (Togo), série Sciences, Tome XVII : 29-48. 